\title{
Fe-doped epitaxial YBCO films prepared by chemical solution deposition
}

\author{
Hong Zhang $\cdot$ Yong Zhao $\cdot$ Wentao Wang • \\ Min Pan · Ming Lei
}

Received: 29 September 2013/Revised: 7 January 2014 / Accepted: 12 January 2014/Published online: 9 February 2014

(C) The Author(s) 2014. This article is published with open access at Springerlink.com

\begin{abstract}
YBa}_{2} \mathrm{Cu}_{3} \mathrm{O}_{7-\delta}(\mathrm{YBCO})$-coated conductors have wide-ranging potential in large-scale applications such as superconducting maglev trains and superconducting electric cables, but low current carrying capability restrains the practical application of YBCO-coated conductors at high temperatures and high magnetic fields. It is crucial to develop YBCO-coated conductors with high critical current density. In this paper, epitaxial, dense, smooth, and crack-free Fe-doped YBCO films were prepared on a $\mathrm{LaAlO}_{3}$ single crystal substrate via a fluorine-free polymerassisted metal organic deposition method. The effects of the dilute $\mathrm{Fe}$ doping on microstructure and superconducting character of YBCO films were investigated. The critical temperature for superconducting of the Fe-doped YBCO films decreases slightly. However, the in-field critical current density of YBCO films improves with dilute Fe doping of amounts less than $x=0.005$, compared to the pure YBCO film. Therefore, the current carrying capability of YBCO film can improve by doping with appropriate amounts of Fe. This means that dilute Fe doping in YBCO films may be a feasible way to prepare high-performance coated conductors.
\end{abstract}

Keywords Epitaxial films - Fe doping - Superconductor . Coated conductor

H. Zhang $(\bowtie) \cdot$ Y. Zhao $(\bowtie) \cdot$ W. Wang $\cdot$ M. Pan · M. Lei Superconductivity and New Energy R\&D Center, Southwest Jiaotong University, Chengdu 610031, China

e-mail: zhanghong@home.swjtu.edu.cn

Y. Zhao

e-mail: yzhao@home.swjtu.edu.cn

\section{Introduction}

Recently, worldwide attention has been paid to the preparation of $\mathrm{YBa}_{2} \mathrm{Cu}_{3} \mathrm{O}_{7-\delta}$ (YBCO)-based coated conductors, i.e., the second generation tapes because of their wideranging potential in large-scale application such as superconducting maglev trains and superconducting electric cables [1-7]. The major approaches for fabricating YBCO-coated conductors include in situ processes like pulse laser deposition (PLD), magnetic sputtering, etc., as well as ex situ wet-chemistry approaches. Chemical solution deposition (CSD) and related techniques provide versatile and cost-effective processing routes with controlled stoichiometry and microstructure [8-12]. Therefore, CSD is a preferred fabrication method. In the early work preparing solution-derived REBCO thin films, the critical current densities were quite limited. It was widely believed that organic-based precursors might lead to the formation of stable $\mathrm{BaCO}_{3}$, which accumulates and precipitates at the grain boundaries (GBs) of REBCO and thus significantly reduces the transport critical current density $J_{\mathrm{c}}$ [13]. In an effort to solve this problem, a promising approach using trifluoroacetates metal organic deposition was developed to deposit YBCO films $[14,15]$. However, the process leads to the formation of HF which is hazardous, and the microstructure of YBCO films were relatively porous [16], which limits the current transport properties. Alternatively, if the formation of $\mathrm{BaCO}_{3}$ can be well controlled, a kind of novel non-fluorine chemical solution deposition method is a promising and costeffective approach to the preparation and application of high performance YBCO films. In this paper, YBCO films were prepared using a non-fluorine chemical solution deposition method which was devised in our laboratory [17-19]. 
The large-scale application of YBCO-coated conductors in superconducting magnets, generators, motors, etc., is prevented because of relatively low current carrying capability with increasing magnetic field and temperature. Therefore, enhancing the $J_{\mathrm{c}}$ is crucial for the practical applications of the YBCO-coated conductors at high temperatures and high magnetic fields. Several methods for increasing the density and effectiveness of vortex pinning sites to enhance $J_{\mathrm{c}}$ were developed by generating artificial defects [20-23]. Studies have confirmed that incorporation of impurities such as $\mathrm{Co}, \mathrm{Fe}, \mathrm{Ga}, \mathrm{Zn}, \mathrm{Ni}$, etc., to the $\mathrm{Cu}$ sites of YBCO can significantly increase the critical current density versus magnetic field $\left(J_{c}-H\right)$ characteristics of high temperature superconductor bulks [24-26]. Dilute impurity doping to the $\mathrm{CuO}$ chain not only enhances flux pinning by lattice deformation but also results in a decrease in critical temperature $\left(T_{\mathrm{c}}\right)$ [24]. There are few reports about the doping effects of these dilute impurities in YBCO films [27, 28], especially those prepared with fluorine-free metal organic deposition (MOD).

The authors have previously published results of cobalt and zinc doping to the copper sites of YBCO [17]. It is a perspective way to essentially improve the current carrying capability of $\mathrm{YBCO}$ film. There is no work of Fe doping of YBCO in their early work. Fe is cheap and easy to obtain, so it is a perspective material for superconductor production. In this study, different dilute quantities of $\mathrm{Fe}$ doping of $\mathrm{YBCO}$ were introduced into the MOD YBCO films. Flux pinning properties of dilute Fe-doped YBCO films were investigated. The films were deposited by a fluorine-free polymer-assisted metal organic deposition (PA-MOD) method [17]. Epitaxial, dense, smooth, and crack-free YBCO films were prepared on a $\mathrm{LaAlO}_{3}$ (LAO) single crystal substrate. In-field $J_{\mathrm{c}}$ of YBCO films was improved by this dilute impurity doping method.

\section{Experimental}

Fe-doped $\mathrm{YBa}_{2} \mathrm{Cu}_{3-x} \mathrm{Fe}_{x} \mathrm{O}_{7-\delta}$ films were deposited on a $\mathrm{LaAlO}_{3}$ (LAO) single crystal substrate using a fluorine-free method [17]. The precursor solutions were synthesized by dissolving acetates of iron, in addition to acetates of yttrium, barium, and copper, in propionic acid with $x=0$, $0.0005,0.001,0.002$, and 0.005 . Then polyvinyl butyral was added into the solution, which was subjected to continuous stirring to adjust the viscosity in order to obtain the final coating solution. The final cation concentration of the solution is $0.6 \mathrm{M}$. Then the solution was coated to LAO using a spin coater with a rotation speed of 6,000 r/min and dried at $150-200{ }^{\circ} \mathrm{C}$ for 5-10 min. The coated samples were fabricated in humid $\mathrm{Ar} / \mathrm{O}_{2}$ mixture gas at $160-500{ }^{\circ} \mathrm{C}$ for $11 \mathrm{~h}$ and then fired at $770-800{ }^{\circ} \mathrm{C}$ in dry $\mathrm{Ar} / \mathrm{O}_{2}$ mixture gas for $1 \mathrm{~h}$. Finally, the samples were annealed in dry $\mathrm{O}_{2}$ gas at $400-450{ }^{\circ} \mathrm{C}$ for $1 \mathrm{~h}$. Finally, the obtained $\mathrm{YBa}_{2} \mathrm{Cu}_{3-x} \mathrm{Fe}_{x} \mathrm{O}_{7-\delta}$-coated films with $x$ of $0-0.005$ were about $500 \mathrm{~nm}$ in thickness.

A Philips X'Pert MRD diffractometer with $\mathrm{Cu}-\mathrm{K} \alpha$ radiation was used to record the $\theta-2 \theta$ X-ray diffraction (XRD) patterns. The microstructure analyses of the YBCO layer were performed using an environmental scanning electron microscope (ESEM). Superconducting transition and magnetic hysteretic loop were measured by Quantum-Design SQUID XL. The $J_{\mathrm{c}}$ value of the YBCO film was determined using Bean critical state model formula.

\section{Results and discussion}

Figure 1 shows the typical $\theta-2 \theta \mathrm{X}$-ray diffraction patterns of $\mathrm{YBa}_{2} \mathrm{Cu}_{3-x} \mathrm{Fe}_{x} \mathrm{O}_{7-\delta}(x=0,0.0005,0.001,0.002,0.005)$ films. As can be seen, only ( $00 l)$ YBCO reflection peaks were detected, excluding the peaks of LAO single crystal substrate. This indicates a strong $c$-axis orientation for all films.

The microstructure analyses of the YBCO layer were performed, and the results are shown in Fig. 2. Dense, smooth, and crack-free surface morphologies can be observed in all the films. A few particles floating on the film surface may be attributed to texture degradation with the growth of film during the firing procedure. We can see the particles in the Fe-doped YBCO films less than the pure YBCO films. There are some very small pores in the

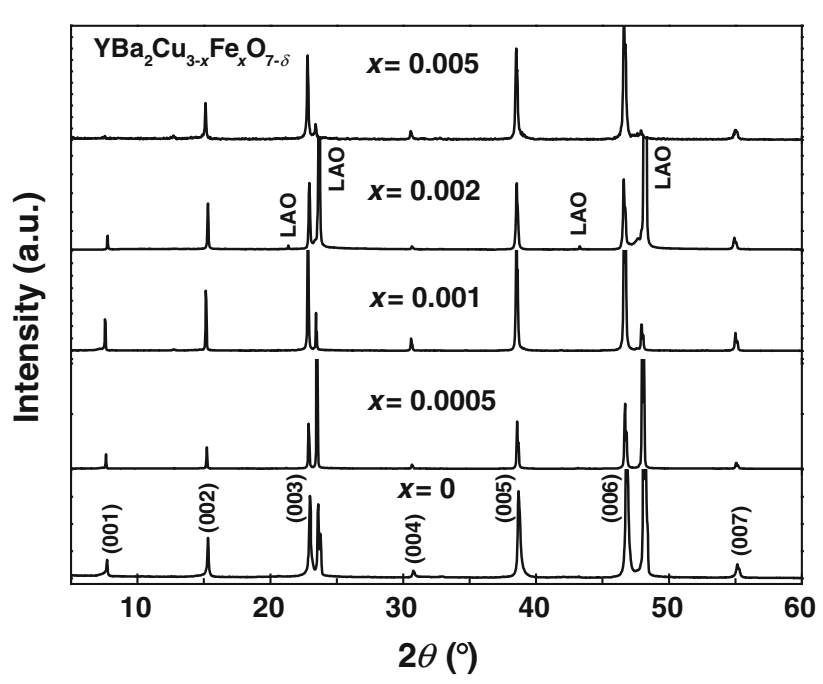

Fig. 1 Typical X-ray diffraction $\theta-2 \theta$ patterns of $\mathrm{YBa}_{2} \mathrm{Cu}_{3-x} \mathrm{Fe}_{x} \mathrm{O}_{7-\delta}$ $(x=0,0.0005,0.001,0.002,0.005)$ films 

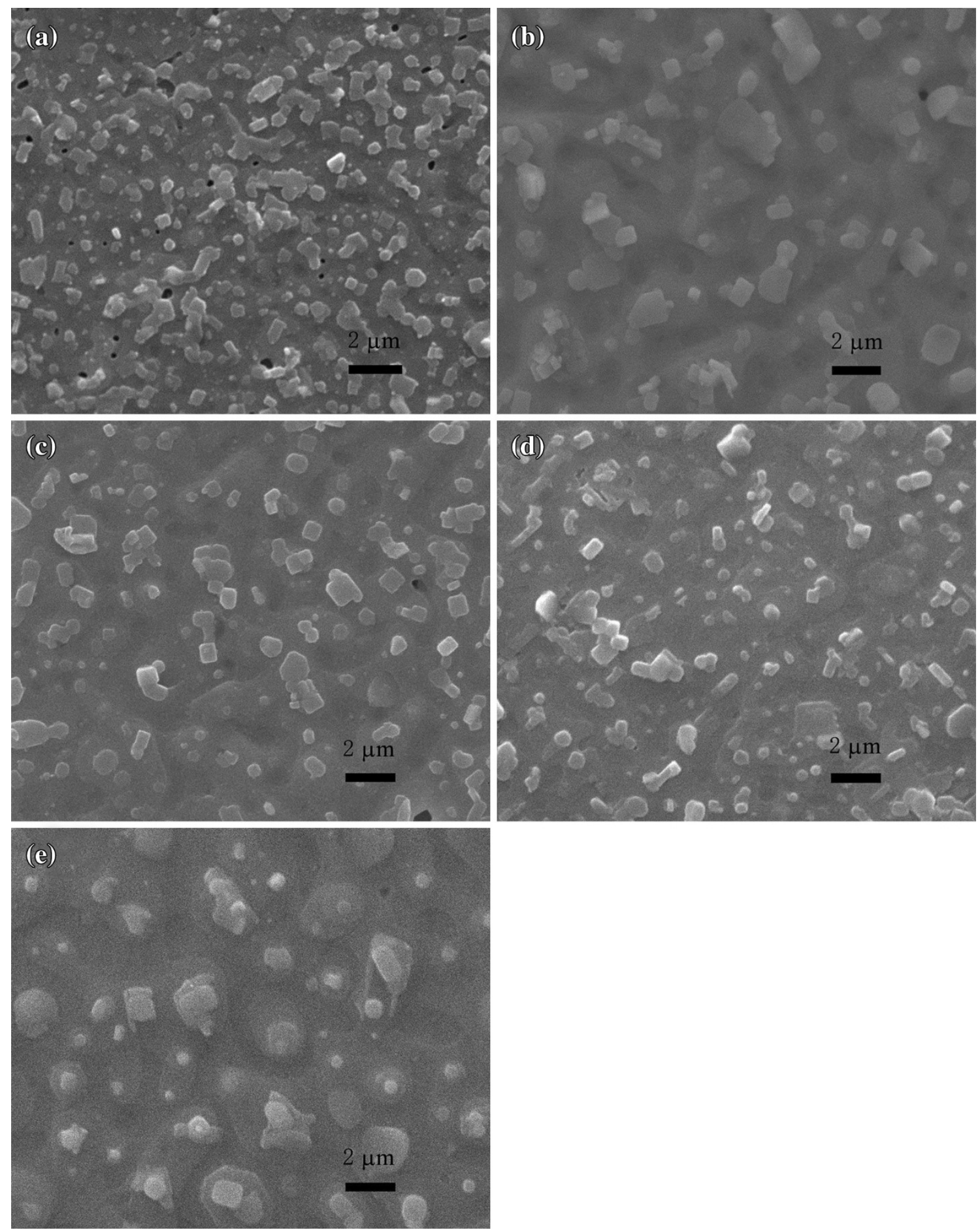

Fig. 2 The surface morphology of $\mathrm{YBa}_{2} \mathrm{Cu}_{3-x} \mathrm{Fe}_{x} \mathrm{O}_{7-\delta}(x=0,0.0005,0.001,0.002,0.005)$ films. a $x=0, \mathbf{b} x=0.0005$, c $x=0.001$, d $x=0.002$, e $x=0.005$

pure YBCO film, but these are not seen in the Fe-doped films. It can be seen that a very small amount of $\mathrm{Fe}$ doping improves the surface connectivity of the YBCO films.
Figure 3 shows the superconductivity critical temperature of $\mathrm{YBa}_{2} \mathrm{Cu}_{3-x} \mathrm{Fe}_{x} \mathrm{O}_{7-\delta}(x=0,0.0005,0.001,0.002$, and 0.005 ) films. The inset shows the temperature dependence of magnetic moment for $\mathrm{YBa}_{2} \mathrm{Cu}_{3-x} \mathrm{Fe}_{x} \mathrm{O}_{7-\delta}$ 


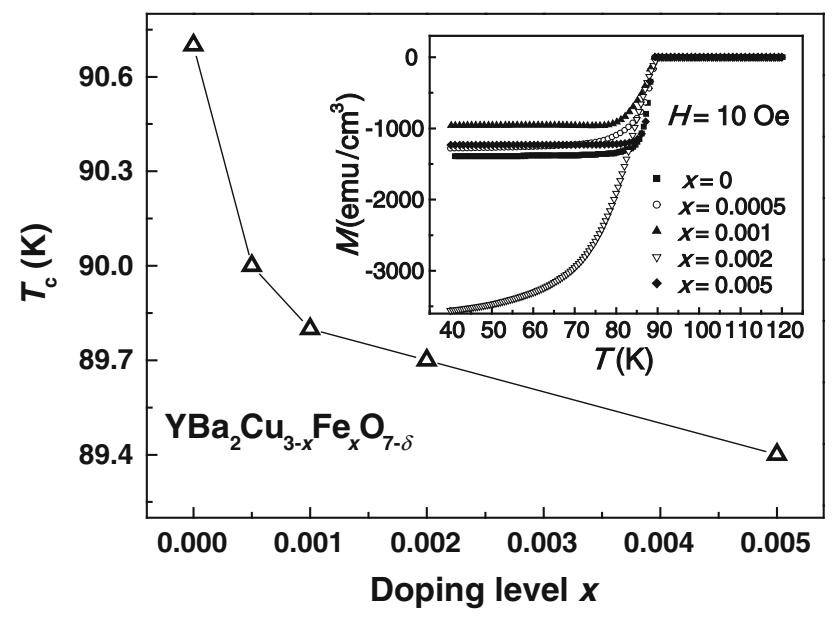

Fig. 3 The superconductivity critical temperature of $\mathrm{YBa}_{2} \mathrm{Cu}_{3-x} \mathrm{Fe}_{x} \mathrm{O}_{7-\delta}(x=0,0.0005,0.001,0.002$, and 0.005) films. Inset is the temperature dependence of magnetic moment for $\mathrm{YBa}_{2} \mathrm{Cu}_{3-x} \mathrm{Fe}_{x} \mathrm{O}_{7-\delta}(x=0,0.0005,0.001,0.002$, and 0.005) films

films. The pure YBCO sample exhibits a sharp superconducting transition; while for the doped samples, the superconducting transition width broadens, showing that such Fe doping leads to a decrease of superconductivity. The decrease may be due to the direct iron substitution for copper in the $\mathrm{Cu}-\mathrm{O}$ chain. The substitution affects the oxygen vacancies which play an important role in determining the transport properties of YBCO. It is found that the superconductivity critical temperature of $\mathrm{YBa}_{2} \mathrm{Cu}_{3-x} \mathrm{Fe}_{x} \mathrm{O}_{7-\delta}$ decreases with $\mathrm{Fe}$ doping. When the value of $x=0.005$ for $\mathrm{YBa}_{2} \mathrm{Cu}_{3-x} \mathrm{Fe}_{x} \mathrm{O}_{7-\delta}$, the $T_{\mathrm{c}}$ value is $1.2 \mathrm{~K}$ smaller than that of pure YBCO film. It exhibits a very small decrease of $T_{\mathrm{c}}$ because of the small amount of Fe doping.

Figure 4 shows the magnetic field dependence of $J_{\mathrm{c}}$ for $\mathrm{YBa}_{2} \mathrm{Cu}_{3-x} \mathrm{Fe}_{x} \mathrm{O}_{7-\delta}(x=0,0.0005,0.001,0.002,0.005)$ films at different temperatures with the magnetic field parallels to the $c$-axis. The $J_{\mathrm{c}}$ values of Fe-doped YBCO films are not significantly higher than that of pure YBCO films at 30 and $50 \mathrm{~K}$. However, at $77 \mathrm{~K}, \mathrm{Fe}$-doped YBCO films possess higher $J_{\mathrm{c}}$ values than those of pure YBCO films, except for the sample with $x=0.005$. As $77 \mathrm{~K}$ is a feasible temperature for application of YBCO superconductors by using liquid nitrogen, this improved $J_{\mathrm{c}}$ value is significant. It means that the current carrying capability of YBCO film can be improved by doping with appropriate amounts of $\mathrm{Fe}$.

\section{Conclusion}

In this paper, from the investigation of the $\mathrm{Fe}$ doping effects on YBCO films, it can be seen that the critical
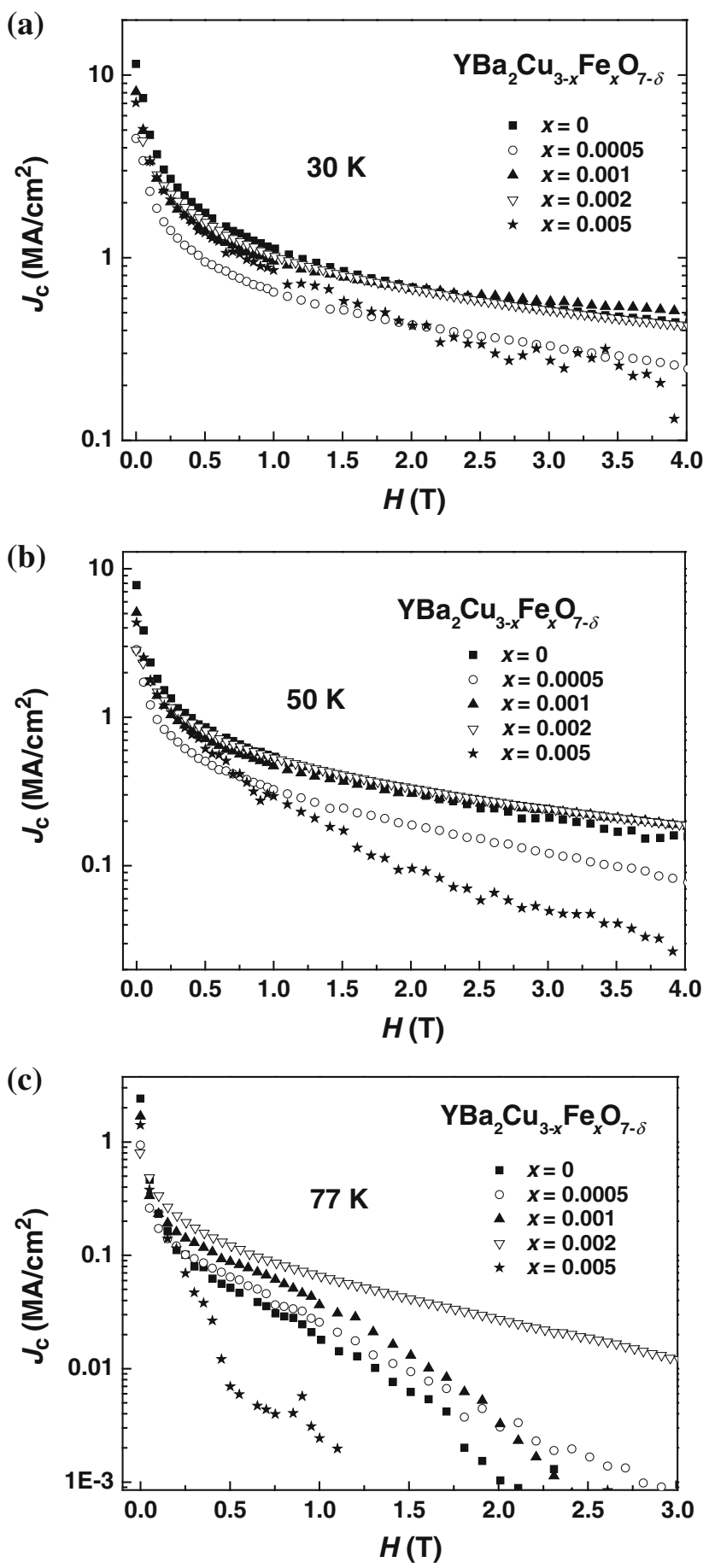

Fig. 4 The magnetic field dependence of critical current densities for $\mathrm{YBa}_{2} \mathrm{Cu}_{3-x} \mathrm{Fe}_{x} \mathrm{O}_{7-\delta} \quad(x=0,0.0005,0.001,0.002,0.005)$ films. a $T=30 \mathrm{~K}, \mathbf{b} T=50 \mathrm{~K}, \mathbf{c} T=77 \mathrm{~K}$

temperature for superconducting decreases slightly, and $J_{\mathrm{c}}$ improves at $77 \mathrm{~K}$ for doping quantities of $x$ less than 0.005 . 
Acknowledgments This research was supported by the Specialized Research Fund for the Doctoral Program of Higher Education (200806131034, 200806130023), Natural Science Foundation of China under Contract Nos. 50672078 and 50872116, the National Science Fund for Distinguished Young Scholars under Contract No. 50588201, and 51102199, the National High-Tech Program of China (863 Program) under Contract No. 2007AA03Z203, the PCSIRT of the Ministry of Education of China (IRT0751), Research and Development Foundation of Southwest Jiao tong University under Grant Contract No. 2004A02, Fundamental Research Funds for the Central Universities (SWJTU12CX019), the National Natural Science Foundation (51202202), and Fundamental Research Funds for the Central Universities of China (SWJTU2682013CX005).

Open Access This article is distributed under the terms of the Creative Commons Attribution License which permits any use, distribution, and reproduction in any medium, provided the original author(s) and the source are credited.

\section{References}

1. Yao X, Oka A, Izumi T, Shiohara Y (2000) Crystal growth and superconductivity of Fe-doped YBCO single crystals. Physica C 339(2):99-105

2. Espinosa-Arronte B, Djupmyr M, Andersson M (2005) Scaling of the $\mathrm{B}$-dependent resistivity for different orientations in $\mathrm{Fe}$ doped $\mathrm{YBa}_{2} \mathrm{Cu}_{3} \mathrm{O}_{7-\delta}$. Physica C 423(1-2):69-73

3. Liu LH, Dong C, Zhang JC, Chen H, Chen L (2002) A simple volumetric method for oxygen content determination in high-Tc doped YBCO compositions. Physica C 383(1-2):17-22

4. Qiao Y, Li Y, Sathiraju S, Reeves J, Lenseth K, Selvamanickam V (2002) An overview of the coated conductor progress at IGCSuperPower. Physica C 382(1):48-51

5. Obradors X, Puig T, Pomar A, Sandiumenge F, Mestres N, Coll M, Cavallaro A, Rom N, Gazquez J, Gonzalez JC, Castano O, Gutierrez J, Palau A, Zalamova K, Morlens S, Hassini A, Gibert M, Ricart S, Moreto JM, Pinol S, Isfort D, Bock J (2006) Progress towards all-chemical superconducting $\mathrm{YBa}_{2} \mathrm{Cu}_{3} \mathrm{O}_{7-y}$ coated conductors. Supercond Sci Technol 19(3):s13-s15

6. Izumi T, Yamada Y, Shiohara Y (2003) Activity of R\&D for HTS coated conductors in Japan. Physica C 392-396(1):9-16

7. Motowidlo LR, Selvamanickam V, Galinski G, Haldar P, Sokolowski RS (2000) Recent progress in high-temperature superconductors at Intermagnetics General Corporation. Physica C 335(1-4):44-50

8. Parans Paranthaman M, Izumi T (2004) High-performance YBCO-coated superconductor wires. MRS Bull 29(9):533-536

9. Parans Paranthaman M (2006) Superconductor wires, McGrawHill 2006 year book of science and technology. McGraw-Hill, New York, pp 319-322

10. Goyal A, Norton DP, Budai JD, Paranthaman M, Specht ED, Kroeger DM, Christen DK, He Q, Saffian B, List FA, Lee DF, Martin PM, Klabunde CE, Hatfield E, Sikka VK (1996) Fabrication of long range, biaxially textured, high temperature superconducting tape on rolled Ni substrates. Appl Phys Lett 69(12): 1795-1797

11. Goyal A, Paranthaman M, Schoop U (2004) The RABiTS approach: using rolling-assisted biaxially textured substrates for high-performance YBCO superconductors. MRS Bull 29(9): 552-561

12. Rupich MW, Verebelyi DT, Zhang W, Kodenkandath T, Li X (2004) Metalorganic deposition of YBCO films for second- generation high-temperature superconductor wires. MRS Bull 29(9):572-578

13. Parmigiani F, Chiarello G, Ripamonti N, Goretzki H, Roll U (1987) Observation of carboxylic groups in the lattice of sintered $\mathrm{Ba}_{2} \mathrm{YCu}_{3} \mathrm{O}_{7-y}$ high- $T_{c}$ superconductors. Phys Rev $\mathrm{B}$ 36(13): $7148-7150$

14. Honjo T, Nakamura Y, Teranishi R, Fuji H, Shibata J, Izumi T, Shiohara Y (2003) Growth mechanism of YBCO films in metal organic deposition method using trifluoroacetates. IEEE Trans Appl Supercond 13(2):2516-2519

15. Minei T, Tanaka T, Ogata M, Mori N, Yamada K, Mukaida M (2006) Growth process and microstructure of Y123 film fabricated by advanced TFA-MOD process. Physica C 445-448(1): $570-573$

16. Castano O, Cavallaro A, Palau A, Gonzalez JC, Rosell M, Puig T, Pinol S, Mestres N, Sandiumenge F, Pomar A, Obradors X (2003) Influence of porosity on the critical currents of trifluoroacetateMOD $\mathrm{YBa}_{2} \mathrm{Cu}_{3} \mathrm{O}_{7}$ films. IEEE Trans Appl Supercond 13(2): 2504-2507

17. Wang WT, Pu MH, Yang Y, Zhang H, Cheng CH, Zhao Y (2010) Enhanced flux-pinning in fluorine-free MOD YBCO films by chemical doping. Physica C 470(20):1261-1265

18. Wang WT, Li G, Pu MH, Sun RP, Zhou HM, Zhang Y, Zhang H, Yang Y, Cheng CH, Zhao Y (2008) Chemical solution deposition of YBCO thin film by different polymer additives. Physica $\mathrm{C}$ 468(15-20):1563-1566

19. Zhao Y, Pu MH, Li G, Du XH, Zhou HM, Zhang YB, Yang XS, Wang Y, Sun RP, Cheng CH (2007) Development of a new series of buffer layers for REBCO coated conductors. Physica C 463-465(1):574-579

20. Feng Y, Pradhan AK, Zhao Y, Wu Y, Koshizuka N, Zhou L (2001) Improved flux pinning in $\mathrm{Y}_{x} \mathrm{Ho}_{1-x} \mathrm{Ba}_{2} \mathrm{Cu}_{3} \mathrm{O}_{y}$ fabricated by powder melting process. Supercond Sci Technol 14(4):224-228

21. Babu NH, Shi YH, Iida K, Cardwell DA (2005) A practical route for the fabrication of large single-crystal (RE)-Ba-Cu-O superconductors. Nat Mater 4(1):476-480

22. Werner M, Sauerzopf FM, Weber HW, Wisniewski A (2000) Fishtail effect in the magnetization of superconducting $\mathrm{RBa}_{2} \mathrm{Cu}_{3} \mathrm{O}_{7-\delta}(\mathrm{R}=\mathrm{Y}, \mathrm{Nd}, \mathrm{Yb})$ and $\mathrm{Y}_{2} \mathrm{Ba}_{4} \mathrm{Cu}_{8} \mathrm{O}_{16}$ single crystals. Phys Rev B 61(21):14795-14803

23. Sauerzopf FM (1998) Anisotropic flux pinning in $\mathrm{YBa}_{2} \mathrm{Cu}_{3} \mathrm{O}_{7-\delta}$ single crystals: the influence of defect size and density as determined from neutron irradiation. Phys Rev B 57(17):10959-10971

24. Ishii Y, Shimoyama JI, Tazaki Y, Nakashima T, Horii S, Kishio $\mathrm{K}$ (2006) Enhanced flux pinning properties of $\mathrm{YBa}_{2} \mathrm{Cu}_{3} \mathrm{O}_{y}$ by dilute impurity doping for $\mathrm{CuO}$ chain. Appl Phys Lett 89(11): 202514-202517

25. Zhou YX, Scruggs S, Salama K (2006) Effects of ionic doping superconducting properties of melt textured $\mathrm{YBa}_{2}\left(\mathrm{Cu}_{1-x} \mathrm{M}_{x}\right)_{3} \mathrm{O}_{7-\delta}$ $(\mathrm{M}=\mathrm{Co}, \mathrm{Ni}, \mathrm{Zn}$ or $\mathrm{Ga}$ ) large grains. Supercond Sci Technol 19(7):S556-S558

26. Pan SH, Hudson EW, Lang KM, Eisaki H, Uchida S, Davis JC (2000) Imaging the effects of individual zinc impurity atoms on superconductivity in $\mathrm{Bi}_{2} \mathrm{Sr}_{2} \mathrm{CaCu}_{2} \mathrm{O}_{8+\delta}$. Nature 403(2):746-750

27. Ohazama T, Mukaida M, Horii S, Saito A, Kita R, Ichinose A, Yoshida Y, Matsumoto K, Ohshima S (2005) Low surface resistance $\mathrm{Zn}$-doped $\mathrm{ErBa}_{2} \mathrm{Cu}_{3} \mathrm{O}_{7-\delta}$ thin films. Physica C 426-431(2):1611-1615

28. Horii S, Ichino Y, Yoshida Y, Matsumoto K, Mukaida M, Ichinose A, Shimoyama J, Kishio K (2006) Dilute Co-doping effects on critical current properties in $\mathrm{PLD}-\mathrm{ErBa}_{2} \mathrm{Cu}_{3} \mathrm{O}_{y}$ films. Physica C 445-448(1):837-840 